\title{
What is the prevalence and risk factors of burnout among pediatric intensive care staff (PICU)? A review
}

\author{
Liz Crowe $^{1,2} \wedge$, Jeanine Young ${ }^{2,3} \wedge$, M. Jane Turner ${ }^{4} \wedge$ \\ ${ }^{1}$ School of Medicine, The University of Queensland, Brisbane, Australia; ${ }^{2}$ School of Nursing, Midwifery and Paramedicine, University of the Sunshine \\ Coast, Sippy Downs, Queensland, Australia; ${ }^{3}$ Centre for Health Services Research, The University of Queensland, Brisbane, Queensland, Australia; \\ ${ }^{4}$ Discipline of Psychiatry, Faculty of Medicine, The University of Queensland, Brisbane, Queensland, Australia \\ Contributions: (I) Conception and design: All authors; (II) Administrative Support: All authors; (III) Provision of study materials: All authors; \\ (IV) Collection and assembly of data: All authors; (V) Data analysis and interpretation: All authors; (VI) Manuscript writing: All authors; (VII) Final \\ approval of the manuscript: All authors. \\ Correspondence to: Liz Crowe. PhD Candidate, School of Medicine, The University of Queensland, Brisbane, Australia. \\ Email: s3346825@student.uq.edu.au; croweliz@bigpond.net.au.
}

\begin{abstract}
Staff in the paediatric intensive care unit work with children and their families in an area of high acuity, mortality, and morbidity. There is complexity due to technological advancements and confronting psychosocial situations. With increasing reports of the threat of burnout to healthcare professionals it is imperative to understand the prevalence of burnout and the determinants of risk factors for staff to work in the paediatric intensive care unit (PICU) in order to inform interventions that reduce risk and support growth and wellbeing of this specialised workforce. We conducted electronic searches of PUBMED, Medline, CINAHL and PsychINFO. Studies meeting eligibility inclusion criteria comprised English text, publication dates 1995 to 2019, use of standardized measures to assess prevalence and risk factors for burnout where the PICU staff data was reported separately and contained sample sizes $\geq 10$ PICU staff. Two reviewers independently identified and extracted citations and assessed the quality of papers using two standardised reporting tools. Twenty studies were included in the final review. Due to the heterogeneity of the included studies a descriptive account of the studies was developed. Outcomes reported included prevalence and levels of burnout reported across professional disciplines, reported scoring criteria for burnout, risk and protective factors for burnout, comparative populations, systems and social context associated with burnout and study strengths and limitations. Most studies were cross-sectional, used a single measure of burnout and focussed on either physicians or nurses. Of the 20 studies reported $62 \%$ reported high burnout, 19\% moderate burnout, and 19\% reported low levels of burnout. Inconsistency was identified in adherence to recommended cut-off scores or reporting for the categorisation of burnout, which contributed to a lack of clarity in the interpretation of prevalence and severity. Reports of factors associated with increased risk and likely protective factors for burnout were often contradictory suggesting that burnout may be situational; dependent upon personal, environmental, leadership, cultural and patient factors within the PICU. This review revealed that determining levels and risk of burnout in PICU staff remains problematic. Further research which examines the experiences of all members of the multidisciplinary team and identification of factors that affect the development of burnout, including those which are protective, is required.
\end{abstract}

Keywords: Pediatric intensive care unit; burnout; psychological; occupational stress

Submitted Nov 24, 2020. Accepted for publication Apr 29, 2021.

doi: $10.21037 / \mathrm{tp}-20-400$

View this article at: http://dx.doi.org/10.21037/tp-20-400

^ ORCID: Liz Crowe, 0000-0002-5270-0389; Jeanine Young, 0000-0003-3849-3392; M. Jane Turner, 0000-0003-1438-217X. 


\section{Background}

The term "burnout" is in popular use in the community and in health settings. It has been defined as "a psychological syndrome emerging as a prolonged response to chronic interpersonal stressors on the job with three key dimensions an overwhelming emotional exhaustion, feelings of cynicism and detachment from the job, and a sense of ineffectiveness and lack of accomplishment" (1) (p103). Burnout is said to occur at an individual level and has been described as a negative psychological experience that involves feelings, attitudes, motives and expectations which create distress, discomfort, dysfunction and negative consequences for the individual however it is not regarded as a medical or psychiatric diagnosis (2). Emotional exhaustion is identified as the core component of burnout (3).

Pediatric intensive care unit (PICU) staff work with children experiencing critical illness and injury; trauma; confronting and tragic psychosocial circumstances; lifelong illnesses and disabilities which may cause moral distress and dilemma; and/or palliation and death. It is therefore intuitive to surmise that there may be high levels of burnout experienced by PICU staff.

Publications reporting burnout in the PICU environment first emerged in the mid-1990s (4). A review by de Cássia Fogaça and colleagues (5) described staff working in pediatric and neonatal intensive care units as 'strong candidates for stress and burnout' and identified the need to develop preventative measures and intervention models.

Despite this call for action there has been little rigorous research on the precise levels of burnout in PICU staff and even fewer studies exploring factors which may be associated with increased risk, or indeed, factors which may be protective. Furthermore, there is some recent dissent from the popular notion of the risk of burnout in intensive care settings; van Mol and colleagues suggested that the issue of burnout remained 'open for discussion' (6).

What is evident is that there are important clinical and health system impacts if health professionals have high levels of burnout. Burnout may adversely impact physical and psychological health of the clinician $(7,8)$ and the individual's professional identity (9) which in turn may negatively impact quality, safety and satisfaction with care, and recovery times of patients $(8,10)$. Ultimately, burnout in the health professional workforce results in organisational issues including poor staff recruitment and retention, job dissatisfaction, poor relationships with colleagues, and staff shortages (11).
Demand for a critical care health professional workforce is projected to grow $(11,12)$. It is therefore imperative to measure contemporary levels of burnout, together with factors associated with both risk and protection, in order to inform interventions that reduce the risk of burnout while supporting the growth and wellbeing of this specialised PICU workforce.

This study systematically reviewed the existing literature to answer three primary research questions: What is the prevalence of burnout in staff working in PICU? Are there identifiable risk factors for the development of burnout in this population? Which factors may be associated with a lower risk of burnout in PICU staff?

\section{Methods}

\section{Data sources and search strategy}

We conducted electronic searches of PUBMED, Medline, CINAHL and PsychINFO, using a combination of keywords and MeSH terms to review the concept of burnout. A university based librarian assisted in, and informed the search strategy, with members of the research team. Keyword search terms included 'pediatric intensive care unit/s', 'PICU', 'pediatrics/paediatrics', 'hospitals', 'burnout', 'professional', 'stress', 'psychological', 'anxiety, professional', 'adaptation, psychological', 'empathy', 'depression', 'stress, occupational', 'fatigue'; 'coping', ‘job satisfaction', 'personal satisfaction', 'job experience', 'stress, psychological', 'stress', 'coping', 'resilience', 'satisfaction', 'emotional exhaustion', 'depersonalisation', 'professional accomplishment', 'retention' and 'environment'. All references were retrieved, organised and stored using Endnote X9. Reference lists of identified articles were hand-searched, supplemented with searches for publications by prominent authors in the field. Eligible studies were those which were (I) primary studies or reviews which examined factors influencing burnout using a quantitative, validate tool and clearly defined methodology, (II) published in peer reviewed journals in the English language; (III) published between January 1, 1995 and December 30, 2019; and which (IV) comprised a staff sample ( $\mathrm{n} \geq 10$ staff) who worked clinically in the PICU. The year 1995 was chosen as the lower limit as it includes the first identified study to investigate burnout in PICU staff (4). While this research (4) is now dated it was included as a comparison to understand if issues have changed or action has been implemented. Studies of mixed staff populations were eligible if the PICU 
staff population data was clearly identifiable and analysed independently. Staff, for the purpose of this review, included any health professional discipline working in PICU as part of the multidisciplinary team. Case reports, dissertations, editorials and commentaries were excluded. Two investigators (LC, JT) reviewed citations independently using a standardised data extraction sheet. A third reviewer (JY) ensured similarity of the two datasets of the initial reviewers. Discrepancies in agreement were resolved by discussion and consensus.

\section{Data synthesis}

Due to the variable nature of the studies and range of assessment measures, it was not possible to combine data into a meta-synthesis. Studies have been presented to illustrate the key features most relevant to study aims. The Strengthening the Reporting of Observational Studies in Epidemiology (STROBE) statement, a 22-item checklist to guide data extraction, ensure adequate reporting, assessment of strengths and weaknesses and discussion was used (13). The Checklist for Prevalence Studies from the suite of Joanna Briggs Institute Critical Appraisal Tools for Systematic Reviews was used to assess the methodological quality of studies reporting prevalence data (14). No papers were excluded because of their validity or quality (See Table 1).

\section{Results}

The initial search identified 1326 citations. Titles and abstracts were checked for relevancy to the topic of staff burnout and duplicates were removed. Abstracts for remaining articles were reviewed and eligibility criteria applied, further narrowing the selection to 57 articles. After full text review, 20 studies met eligibility criteria for inclusion in the review (See Figure 1). Studies were excluded due to PICU staff samples of less than 10 participants, PICU staff data was not clearly identifiable in a mixed staff cohort, or burnout was not measured using a validated, quantitative tool.

\section{Characteristics of included studies}

All studies were published in English. The earliest study was published in 1995 (4); remaining studies were published from 2010 to 2019. North American studies predominated $(\mathrm{n}=10,50 \%)(4,15-23)$ while Europe $(\mathrm{n}=4,20 \%)$ included one study each originating from Turkey (24), Greece (25),
Spain (26) and Germany (10). United Kingdom ( $\mathrm{n}=2,10 \%)$ $(27,28)$, South America $(\mathrm{n}=2,10 \%)(29,30)$ and single studies from Canada (5\%) (31) and Asia (5\%) (32) were also represented. No studies meeting eligibility criteria originated from Australasia. Table (https://cdn.amegroups. $\mathrm{cn} /$ static/public/tp-20-400-1.pdf) provides summary information from each of the studies.

This review included observational studies predominantly using cross-sectional survey designs $(n=18)$ to investigate levels of burnout within health professional populations. Of the two remaining studies examining burnout in PICU staff populations, one used a pre and post-implementation design with the intervention being interactive, facilitated peer support sessions (18). The second implemented a longitudinal design using a cross-sectional survey administered at two time points following rotation of medical residents in the PICU (22).

Study sample sizes ranged from 14 to 1656 participants, across 20 studies, which reported burnout from PICU health professional populations (total $n=5,381$ ). PICU staff populations studied for burnout included physicians $(\mathrm{n}=8$ studies, $\mathrm{n}=2,704)(4,10,19,21-23,29,30)$, nurses ( $\mathrm{n}=6$ studies, $\mathrm{n}=392)(15,17,20,24,31,32)$; and physicians and nurses combined ( $\mathrm{n}=3$ studies, $\mathrm{n}=515)(25-27)$. The remaining studies $(\mathrm{n}=3)$ included allied health professionals and the wider PICU team $(n=1,770)(16,18,28)$. Nine studies focussed on burnout experienced by staff solely in the PICU environment $(4,18,19,21-23,28,29,32)$; the remaining studies $(n=11)$ reported results from the PICU population as a subset of a wider health professional study examining burnout and/ or other phenomena, such as compassion fatigue.

Of those studies that reported age and clinical experience, the mean age of study participants was approximately 35 years (range 18 years $\geq 60$ years) while clinical experience within PICU varied considerably (range less than 1 year to $\geq 30$ years).

A range of validated self-report measures were used to examine burnout in eligible studies. The most frequently used tool was the Maslach Burnout Inventory (MBI) ( $\mathrm{n}=13$ studies) (33) inclusive of the 22-item MBI $(\mathrm{n}=10)$ (10,17,21,24-26,29-31); and the abbreviated 9-item MBI $(\mathrm{n}=3)(19,27,28)$. The Professional Quality of Life version 5 (34) $(\mathrm{n}=3)(15,16,20)$, the Aronson and Pine Burnout Scale (1981) (4,35), the Occupational Burnout Inventory (adapted from the Copenhagen Burnout Inventory and translated) $(32,36)$; the Copenhagen Burnout Inventory $(18,36)$, and the Compassion Satisfaction Self-Test for Helpers 1995 $(23,37)$ were also used to score levels of burnout in selected studies. See Table (https://cdn.amegroups.cn/static/public/ 


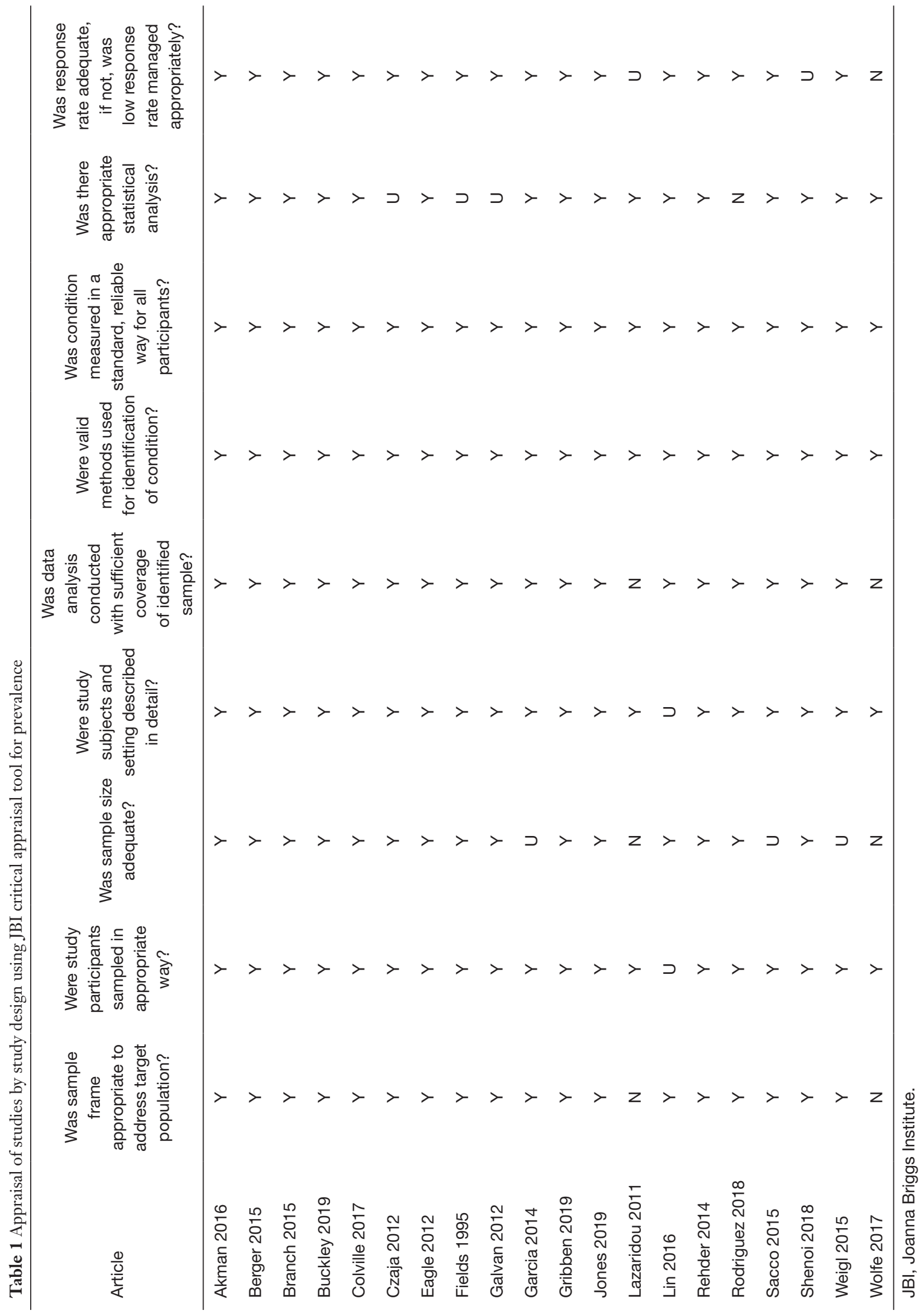




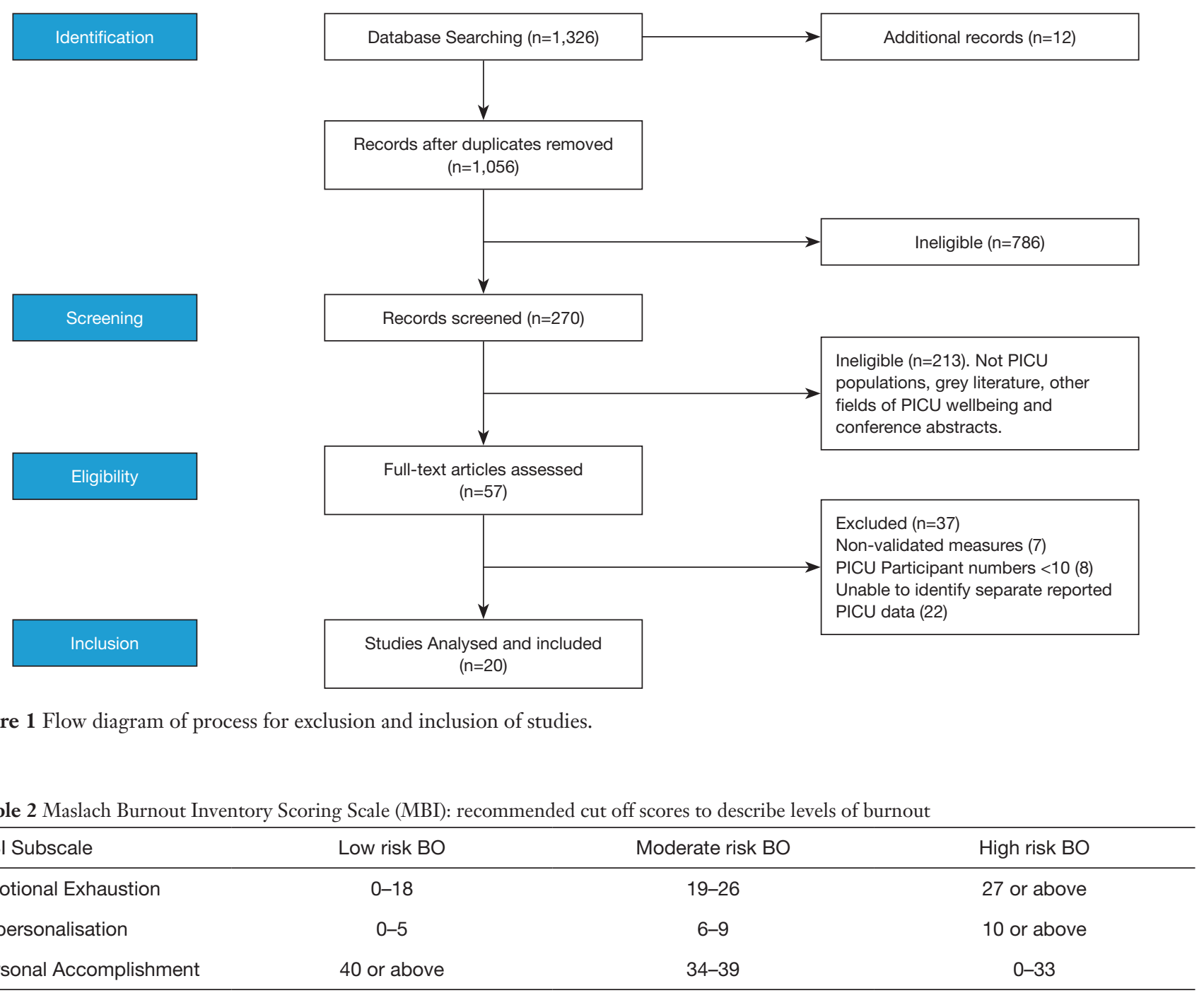

tp-20-400-1.pdf) and Table 2. The MBI is the most widely used and validated tool to measure burnout and is regarded in the literature as the 'gold standard' in the measurement of burnout $(38,39)$.

This review examines burnout experienced by PICU staff discussed under the broad headings of: prevalence, sociodemographic characteristics, factors associated with high and low risk of burnout, comparison of burnout in PICU with other health settings, and systems and social contextual factors contributing to burnout.

\section{Prevalence of burnout}

Interpretation of the prevalence of burnout in PICU staff is complicated due to the range of measures, variations in scoring and approach to data analyses and interpretation. Lowest prevalence in eligible studies was reported in a small sample $(\mathrm{n}=33)$ of North American nurses using ProQOLv5: high burnout (1\%), moderate $(32 \%)$ and low burnout $(67 \%)(20)$. In contrast, the highest prevalence of burnout was documented in a small sample of PICU physicians from Brazil $(n=35)$ in which $63 \%$ experienced high burnout scores measured by the 22 item MBI (30). A large contemporary study $(\mathrm{n}=1,656)$ including only PICU interdisciplinary staff from the United Kingdom reported high burnout, identifying junior doctors as being of notable risk (28). In the larger studies, the determinants, tools and scoring are too diverse or unclear to draw 
conclusions.

\section{Sociodemographic characteristics and risk of burnout}

Age was the variable most frequently associated with burnout; however, results demonstrate inconsistencies. Higher burnout scores have been associated with younger age $(15,24,28)$, older age $(17,20,21)$ while several eligible studies $(n=4)$ reported no association with burnout and age $(16,25-27)$.

Gender and association with burnout are less frequently reported. Female physicians were reported to experience higher rates of emotional exhaustion (MBI burnout component) than male colleagues in two studies $(4,21)$. Although Shenoi and colleagues found emotional exhaustion higher in females compared to males ( $42 \% v s$. 29.4\%), when "severe burnout" was examined, this gender difference was no longer apparent (21). No other studies reported gender to be an association.

Relationship and parenting status were variably reported to be associated with burnout, with inconsistencies in results apparent. One study reported that single relationship status was associated with increased risk of burnout $(\mathrm{P}<0.01)(29)$, while another found being married was protective against burnout (24). Other studies with varied sample sizes (range $n=14-275$ ) have not identified relationship status as being significantly associated with burnout $(21,25,27)$, despite all utilising the MBI as the measurement tool. Although one study with a moderate sample size $(\mathrm{n}=162)$ reported that having children was protective against burnout $(\mathrm{P}<0.003)(29)$, other studies reported no association $(21,24,27,30)$.

A range of professional factors were reported to be associated with high scores for burnout, however, inconsistencies in findings across studies were evident. The contribution of professional discipline as a determinant in risk of burnout remains unclear with inconsistent results reported across studies. Nurses reported significantly higher emotional exhaustion (EE) in two studies using both 9 and 22 -item versions of the MBI $(25,28)$. Lazaridou et al. (25) also reported higher depersonalisation (DP) scores for nurses compared to physicians in their sample (total $\mathrm{n}=66$ including 14 PICU staff); however in contrast a much larger PICU focused sample $(n=1,656)$ recruited by Jones and colleagues (28) using the abbreviated MBI, reported physician DP scores to be significantly higher than nurses (28). Findings from Colville and colleagues (2017) were consistent with Jones et al., with physicians twice as likely to experience significantly higher burnout scores than compared to nurses (27).

Determining if experience across the trajectory of a PICU career has points where burnout is more prevalent is also difficult to establish, particularly given the crosssectional nature of these studies which does not allow for experience of burnout to be evaluated against actual career choices. Of studies examining this association, most did not report a significant association between experience and/or seniority and burnout (16,24-27,31). While three studies identified a significantly increased risk for junior versus senior physicians $(10,28)$ and less experienced nurses (PICU experience 4-10 years $v s$. more than 10 years) (15), only one older study by Field and colleagues reported that increased years of practice was associated with higher burnout in physicians (4). This study by Fields and colleagues was the only study to use the Aronson and Pine Burnout Scale and may not be reflective of contemporary physician experiences given data collection occurred over 25 years ago.

Environmental and employment characteristics were reported as associated with high burnout. Shift work, night duty and time spent in the hospital were each significantly associated with increased burnout for physicians $(10,19)$. Physical work environment and interprofessional relationships were also examined in several studies. Physical capacity of the PICU in terms of bed numbers and occupancy was correlated to increased burnout risk $(\mathrm{P}<0.01)$ in a large interprofessional study $(\mathrm{n}=1,656)(28)$. PICUs with mixed paediatric populations, high acuity patients and high patient to staff ratios were also found to be factors for associated with higher staff burnout in three studies $(16,21,24)$. Cultural factors within PICU units that were identified as being associated with high burnout included: conflict with colleagues $(23,26)$; the staff member not feeling valued by colleagues (4), inadequate staffing or perceived unfairness in staffing levels (10), high administration demands (23) and change in management or leadership structure in the past twelve months (20). Venting emotion and using alcohol were each associated with a doubling in the risk of reported burnout in a British study of health professionals, conducted by Colville and colleagues (27).

Several studies reported associations that were either protective or resulted in lower burnout although these findings were not universal. An inverse relationship between regular exercise and burnout was reported in two studies; however what constituted 'regular' was not clearly defined $(4,21,27)$. Perceptions of oneself being a valued member of the PICU team was protective in several 
studies $(4,17)$, as was a strong sense of overall competency in skill level (32). Positive attitudes about engaging with patients and families were associated with perceptions of greater personal accomplishment and overall lower burnout, despite moderate emotional exhaustion scores (31). Direct relationships were also identified for several variables already described; lower burnout scores were associated with working less nights and less hours with time disengaged from the PICU, particularly where there was a Fellow or senior physician to support junior staff on all shifts (19). PICU units that had one area level of specialty, for example a paediatric cardiac PICU, reported less burnout than the PICUs who accepted all critically ill patients (20). The opportunity for staff to be involved in debriefing after a critical incident was also associated with lower burnout, with reports that the risk for burnout was halved (27).

While there were no multinational studies identified in the eligible literature, use of standardised cut-off scores for validated burnout tools allows comparison of burnout between countries. PICU staff from developing countries consistently reported higher burnout scores than PICU staff working in developed nations; high burnout identified in studies arising from developing nations was predominantly ascribed to poor working conditions $(25,29)$.

\section{Comparison of rates of burnout between PICU staff and other staff populations}

Eleven of the studies reported in this review comprised of samples contributing to broader studies examining burnout in healthcare settings which allowed comparisons between staff populations. When compared to adult critical care and general acute care settings, PICU staff were reported to be at higher risk of burnout in five studies $(17,25,27,30,31)$, equivalent risk in one study (26), while a similar proportion of studies $(n=5)$ reported a lower incidence of risk of burnout between PICU staff when compared to health settings $(10,15,20,24,31)$. Most of these studies ( $n=8$ of $11,73 \%$ ) consistently used versions of the MBI, while the remaining studies used the ProQOL to measure burnout $(\mathrm{n}=3,17 \%)$; however, PICU sample sizes varied from 14-298 participants.

\section{Additional factors which may impact burnout—systems and social context}

The twenty studies included in this review were conducted in diverse cultural and health settings with disparate resources resulting in an inability to generalise across PICU settings globally. Results are not intuitive with no specific trends identified. High burnout was reported in all studies conducted in developing countries and was generally ascribed to poor working conditions with high patient ratios/understaffing $(24,25)$, suboptimal wages $(24,25,29)$, increased on-call demands $(29,30)$, and no permanent staff contracts (29).

\section{Factors which may be associated with lower or no burnout}

Several studies reported associations that were either protective or resulted in lower burnout although these findings were not universal. Perceptions of oneself as being a valued member of the PICU team and the ability to work in a positive environment was protective for individuals in several studies $(4,17)$, as was a strong sense of overall competency in skill level (32). There was consensus that having reduced rates of on-call, working less nights and time disengaged from the PICU was helpful in reduction of burnout $(4,10,19)$.

\section{Limitations of the data}

Interpretation of the data is complex because of several methodological issues. Sample sizes vary and are particularly small in the comparative populations for which PICU participants were a subset of a wider study. Six tools were used to measure burnout across the studies, there is heterogeneity within populations, health settings, patient/nurse ratios, working hours, conditions, and rates of pay which makes results in determination of accurate estimations of burnout prevalence and identification of risk factors challenging. There are inconsistencies and failure to report cut-off scores, with studies frequently not defining cut off scores or modifying scores confusing what constitutes a score of 'burnout'. The Maslach Burnout Inventory (MBI) was the most frequently used measure ( $\mathrm{n}=13 / 20$ studies). Several studies reported burnout based solely on high scores in either the subscales of Emotional Exhaustion (EE) or Depersonalisation (DP) $(15,27,28)$ without recognition that the original MBI stated that two or more of the subscales needed to identify high scores for burnout to be a concern $(1,40)$. Two studies used different cut-offs for scoring the MBI and thus reported higher burnout than would be reported using the recommended cut-off scores $(26,29)$. One study reported percentages of 
respondents categorised as 'no burnout syndrome' or 'any burnout' without reporting raw data or actual scores in each subscale (21). The ProQOL uses a scale of burnout to determine compassion fatigue though several studies report on burnout independently.

Most studies were cross-sectional; reported associations with burnout do not infer causality. Low response rates and small sample sizes evident in several studies contributed to methodological limitations. Inclusion of single studies from some countries is a further limitation. Eleven studies conducted research solely in the PICU. Ten studies conducted with mixed staff settings presented composite demographic characteristics rather than reporting on PICU staff as a subset, so results may not be generalisable or applicable to the PICU population. Several studies provided limited or no demographic data $(18,19,31)$ and most studies were conducted in Western industrialised settings where health systems and models of health care vary considerably from developing nations.

Only three of the studies had a population sample representative of the PICU interdisciplinary team despite recognition that interdisciplinary teamwork is critical to PICU patient care and outcomes (41).

\section{Discussion}

The primary aim of this review was to determine the prevalence of burnout in PICU staff and identify risk and protective factors. Prevalence of burnout ranged from $1 \%$ to $67 \%$ across the twenty eligible studies. Results across studies are inconsistent making clear identification of risk and protective factors difficult. Variations in measurement and scoring for burnout and reporting of results across populations and within subsets of populations, limits comparisons and generalisability of results. This is a common theme in the burnout literature $(40,42)$.

Understanding the implications of burnout in the PICU is challenging. Studies have found inconclusive evidence about compassion fatigue and burnout in the adult intensive care setting (6) and the prevalence of burnout among physicians respectively (43). These findings may relate in part to the issue of measurement. Authors of the MBI state that neither the coding nor the original numerical scores should be used for diagnostic purposes of burnout as there is insufficient research on the pattern(s) of scores as indicators of individual dysfunction or the need for intervention (1,40). In 2018, Knox and colleagues compared the Maslach Burnout Inventory with a self-defined single- item burnout measure and found that prevalence of burnout differed considerably depending on the survey instrument and that the MBI has a higher sensitivity than a self-defined measure (44). The most recent MBI recommendation proposes that burnout should no longer be measured as a dichotomy (burnout/no burnout) but rather as a continuum of experience that is represented as a profile; however this approach has not yet been operationalised (40).

The increased focus on burnout has caused some to consider if burnout may be "contagious" $(45,46)$. Bakker et al in 2005 surveyed 1849 intensive care nurses across 80 intensive care units in 12 European countries and found that prevalence of burnout was consistent in individual intensive care units and complaints of burnout in the team was the most important predictor of burnout for individuals and units even after controlling for other factors (46). Single centre studies may be influenced by organisational philosophy and leadership as well as emotional contagion. Emotional contagion is a well-researched phenomenon particularly in the work context with increased interest in the relationship to burnout in the health setting (47-49). It is unknown if social media has impacted the prevalence and severity of burnout within the critical care population (50-52) as social media now plays an important role in medical and nursing education within critical care (53-55).

It is timely to review 'burnout' within PICU in a broader social context as burnout is increasingly being recognised as a multi-faceted socio-cultural phenomenon (56). Burnout occurs across occupations as individuals are constantly challenged with a 'persistent imbalance of demands over resources' (57) (p208) in relation to energy and time particularly at work, with employees often holding personal values that differ from organisations' (57). A further source of frustration and burnout may be that hospitals and health services articulate ideals that far exceed their resources notwithstanding the best intentions of their staff (57). Development of burnout in individuals is likely to be multidimensional and largely influenced by systems and their interactions with personal factors (58). Maslach and Leiter, recognised as seminal authors of burnout, have purported that burnout research has been oversimplified and used as a diagnosis, disability or disease which was never the intention, and for which there is no clinical evidence (40). They describe a new scoring procedure to bring together all three MBI dimensions in a comprehensive and meaningful way, and have generated five profiles to characterise an individual's work experience: burnout, overextended, ineffective, disengaged, and engagement (40).

This review highlights the limitations of the use of cross- 
sectional measures to establish prevalence and risk factors of burnout. These measures fail to systematically examine ecological factors such as working conditions, team culture, leadership, the working environment, and organisational issues likely to affect health professionals. Although validated, these tools do not concurrently measure personal and employment conditions, or social and cultural factors, that contribute to stress and distress in an individual's life. Perhaps more critically, none of the 20 articles included in this review have explored the implications of their findings for the individuals' health or tangible outcomes for patients or the team culture.

\section{Conclusions}

The findings of this review are consistent with reported findings of systematic reviews on burnout in the adult intensive care setting (6) and for physicians (43). Deficiencies in the current literature relating to burnout, specifically the absence of multidimensional approaches to identify burnout and promote wellbeing of the PICU staff, were identified. Future research on burnout experienced by PICU staff should be inclusive of the suggested profiles proposed in the recently revised Maslach Burnout Inventory (40) rather than using a binary burnout/no burnout model. Without accurate measures of the phenomenon of burnout together with related risk and protective factors of burnout in the PICU, the development and implementation of effective interventions, and subsequent mitigation of burnout symptoms, is challenging. Ecological or organisational systems models which offer approaches to understand the complex systems in which burnout may manifest (59), and to identify and manage burnout experienced by health professionals, may be useful to inform supportive strategies for staff working in the unique work environment of PICU into the future.

\section{Acknowledgments}

Funding: None.

\section{Footnote}

Provenance and Peer Review: This article was commissioned by the Guest Editors (Jan Hau Lee, Vijay Srinivasan, and Debbie Long) for the series "Pediatric Critical Care" published in Translational Pediatrics. The article has undergone external peer review.
Peer Review File: Available at http://dx.doi. org/10.21037/tp$20-400$

Conflicts of Interest: All authors have completed the ICMJE uniform disclosure form (available at http://dx.doi. org/10.21037/tp-20-400). The series "Pediatric Critical Care" was commissioned by the editorial office without any funding or sponsorship. The authors have no conflicts of interest to declare.

Ethical Statement: The authors are accountable for all aspects of the work in ensuring that questions related to the accuracy or integrity of any part of the work are appropriately investigated and resolved.

Open Access Statement: This is an Open Access article distributed in accordance with the Creative Commons Attribution-NonCommercial-NoDerivs 4.0 International License (CC BY-NC-ND 4.0), which permits the noncommercial replication and distribution of the article with the strict proviso that no changes or edits are made and the original work is properly cited (including links to both the formal publication through the relevant DOI and the license). See: https://creativecommons.org/licenses/by-nc-nd/4.0/.

\section{References}

1. Maslach C, Leiter MP. Understanding the burnout experience: recent research and its implications for psychiatry. World Psychiatry 2016;15:103-11.

2. Maslach C. Measuring burnout. The Oxford handbook of organizational well-being. 2008:86-108.

3. Seidler A, Thinschmidt M, Deckert S, et al. The role of psychosocial working conditions on burnout and its core component emotional exhaustion - a systematic review. J Occup Med Toxicol 2014;9:10.

4. Fields AI, Cuerdon TT, Brasseux CO, et al. Physician burnout in pediatric critical care medicine. Crit Care Med 1995;23:1425-9.

5. Fogaça MC, de Carvalho B, de Albuquerque Cítero V, et al. Factors that cause stress in physicians and nurses working in a pediatric and neonatal intensive care unit: bibliographic review. Revista Brasileira de Terapia Intensiva 2008;20:261-6.

6. van Mol MM, Kompanje E, Benoit D, et al. The prevalence of compassion fatigue and burnout among healthcare professionals in intensive care units: a systematic review. PLoS One 2015;10:e0136955. 
7. Patel RS, Bachu R, Adikey A, et al. Factors Related to Physician Burnout and Its Consequences: A Review. Behav Sci (Basel) 2018;8:98.

8. West CP, Dyrbye LN, Shanafelt TD. Physician burnout: contributors, consequences and solutions. J Intern Med 2018;283:516-29.

9. Schaufeli WB. Burnout, Fatigue, Exhaustion: An Interdisciplinary Perspective on a Modern Affliction. In: Necknna S, Schaffner A, Wagner G, editors. Burnout, Fatigue, Exhaustion: An Interdisciplinary Perspective on a Modern Affliction. 1st ed. Germany: Springer Verlag; 2017. p. 105-27.

10. Weigl M, Schneider A, Hoffmann F, et al. Work stress, burnout, and perceived quality of care: a cross-sectional study among hospital pediatricians. Eur J Pediatr 2015;174:1237-46.

11. Reith TP. Burnout in United States Healthcare Professionals: A Narrative Review. Cureus 2018;10:e3681.

12. Khanna AK, Majesko AA, Johansson MK, et al. The Multidisciplinary Critical Care Workforce: An Update from SCCM: Society of Critical Care Medicine; 2019 Available online: https://www.sccm.org/Communications/ Critical-Connections/Archives/2019/TheMultidisciplinary-Critical-Care-Workforce-An.

13. von Elm E, Altman DG, Egger M, et al. The Strengthening the Reporting of Observational Studies in Epidemiology (STROBE) statement: guidelines for reporting observational studies. Ann Intern Med 2007;147:573-7.

14. Institute TJB. JBI Critical Appraisal Tools: Checklist for Prevalence Studies Adelaide: The University of Adelaide; 2017 [cited 2020. Available online: http://joannabriggs. org/research/critical-appraisal-tools.html.

15. Berger J, Polivka B, Smoot EA, et al. Compassion Fatigue in Pediatric Nurses. J Pediatr Nurs 2015;30:e11-7.

16. Branch C, Klinkenberg D. Compassion Fatigue Among Pediatric Healthcare Providers. MCN, The American Journal of Maternal/Child Nursing 2015;40:160-6.

17. Czaja AS, Moss M, Mealer M. Symptoms of posttraumatic stress disorder among pediatric acute care nurses. J Pediatr Nurs 2012;27:357-65.

18. Eagle S, Creel A, Alexandrov A. The effect of facilitated peer support sessions on burnout and grief management among health care providers in pediatric intensive care units: a pilot study. J Palliat Med 2012;15:1178-80.

19. Rehder KJ, Cheifetz IM, Markovitz BP, et al. Pediatric Acute Lung I, Sepsis Investigators N. Survey of in-house coverage by pediatric intensivists: characterization of 24/7 in-hospital pediatric critical care faculty coverage. Pediatr Crit Care Med 2014;15:97-104.

20. Sacco TL, Ciurzynski SM, Harvey ME, et al. Compassion satisfaction and compassion fatigue among critical care nurses. Crit Care Nurse 2015;35:32.

21. Shenoi AN, Kalyanaraman SM, Pillai SA, et al. Burnout and Psychological Distress Among Pediatric Critical Care Physicians in the United States*. Crit Care Med. 2018;46:116-22.

22. Wolfe KK, Unti SM. Critical care rotation impact on pediatric resident mental health and burnout. BMC Med Educ 2017;17:181.

23. Gribben JL, Kase SM, Waldman ED, et al. A CrossSectional Analysis of Compassion Fatigue, Burnout, and Compassion Satisfaction in Pediatric Critical Care Physicians in the United States. Pediatr Crit Care Med 2019;20:213-222.

24. Akman O, Ozturk C, Bektas M, et al. Job satisfaction and burnout among paediatric nurses. J Nurs Manag 2016;24:923-33.

25. Lazaridou C, Agakidou E, Diamanti E, et al. Burnout in doctors and nurses working in neonatal and pediatric intensive care units in a General Hospital. Aristotle University Medical Journal 2011;38:37-41.

26. Rodríguez-Rey R, Palacios A, Alonso-Tapia J, et al. Burnout and posttraumatic stress in paediatric critical care personnel: Prediction from resilience and coping styles. Aust Crit Care 2019;32:46-53.

27. Colville GA, Smith JG, Brierley J, et al. Coping With Staff Burnout and Work-Related Posttraumatic Stress in Intensive Care. Pediatr Crit Care Med 2017;18:e267-e273.

28. Jones GAL, Colville GA, Ramnarayan P, et al. Psychological impact of working in paediatric intensive care. A UK-wide prevalence study. Arch Dis Child 2020;105:470-5.

29. Galván ME, Moreno RP, Sarli M, et al. Professional burnout in pediatric intensive care units in Argentina. Archivos Argentinos de Pediatría 2012;110:466-73.

30. Garcia TT, Garcia PCR, Molon ME, et al. Prevalence of burnout in pediatric intensivists: an observational comparison with general pediatricians. Pediatr Crit Care Med 2014;15:e347.

31. Buckley L, Christian M, Gaiteiro R, et al. The relationship between pediatric critical care nurse burnout and attitudes about engaging with patients and families. The Canadian Journal of Critical Care Nursing 2019;30:22-8.

32. Lin TC, Lin HS, Cheng SF, et al. Work stress, occupational burnout and depression levels: a clinical study 
of paediatric intensive care unit nurses in Taiwan. J Clin Nurs 2016;25:1120-30.

33. Maslach C, Jackson S, Leiter MP, et al. Maslach Burnout Inventory Manual 3rd Edition. 3rd ed: Mind Garden; 1996.

34. Stamm BH. The Concise ProQOL Manual. 2nd ed: Pocatello; 2010.

35. Pines A, Aronson E, Kafry D. Burnout: From Tedium to Personal Growth. New York: The Free Press, Division of Macmillan; 1981.

36. Kristensen TS, Borritz M, Villadsen E, et al. The Copenhagen Burnout Inventory: A new tool for the assessment of burnout. Work Stress 2005;19:192-207.

37. Figley CR. Compassion fatigue: coping with secondary traumatic stress disorder in those who treat the traumatized. New York: Brunner/Mazel; 1995.

38. Pantaleoni JLMD, Augustine EMMD, Sourkes BMP, et al. Burnout in Pediatric Residents Over a 2-Year Period: A Longitudinal Study. Acad Pediatr 2014;14:167-72.

39. Williamson K, Lank PM, Cheema N, et al. Comparing the Maslach Burnout Inventory to Other Well-Being Instruments in Emergency Medicine Residents. J Grad Med Educ 2018;10:532-6.

40. Maslach C, Leiter MP. How to Measure Burnout Accurately and Ethically. Harvard Business Review 2021:7.

41. Stocker M, Pilgrim S, Burmester M, et al. Interprofessional team management in pediatric critical care: some challenges and possible solutions. J Multidiscip Healthc 2016;9:47-58.

42. Eckleberry-Hunt J, Kirkpatrick H, Barbera T. The Problems With Burnout Research. Acad Med 2018;93:36770.

43. Rotenstein LS, Torre M, Ramos MA, et al. Prevalence of burnout among physicians: A systematic review. JAMA 2018;320:1131-50.

44. Knox M, Willard-Grace R, Huang B, et al. Maslach Burnout Inventory and a Self-Defined, Single-Item Burnout Measure Produce Different Clinician and Staff Burnout Estimates. J Gen Intern Med 2018;33:1344-51.

45. González-Morales MG, Peiró JM, Rodríguez I, et al. Perceived collective burnout: a multilevel explanation of burnout. Anxiety Stress Coping 2012;25:43-61.

46. Bakker AB, Le Blanc PM, Schaufeli WB. Burnout contagion among intensive care nurses. J Adv Nurs 2005;51:276-87.

47. Bakker A, van Emmerik H, Euwema MC. Crossover of Burnout and Engagement in Work Teams. Work Occup 2006;33:464-89.
48. Schaefer RAB, Palanski ME. Emotional Contagion at Work: An In-Class Experiential Activity. J Manage Educ 2014;38:533-59.

49. Petitta L, Jiang L, Härtel CEJ. Emotional contagion and burnout among nurses and doctors: Do joy and anger from different sources of stakeholders matter? Stress and Health 2017;33:358-69.

50. Ferrara E, Yang Z. Measuring Emotional Contagion in Social Media. PLoS One 2015;10:e0142390.

51. Cui X. Emotional Contagion or Symbolic Cognition? A Social Identity Perspective on Media Events. J Broadcast Electron 2018;62:91-108.

52. Kramer AD, Guillory J, Hancock J. Experimental evidence of massive-scale emotional contagion through social networks. Proc Natl Acad Sci U S A 2014;111:8788-90.

53. Barnes S, Riley C, Kudchadkar S. 416: Social Media for dissemination of pediatric critical care content: A Hashtag Analysis. Crit Care Med 2016;44:A180.

54. Depriest A, Harwayne-Gidansky I, Gerlach A, et al. Improving Multidisciplinary Involvement At The Critical Care Congress Through Social Media. Crit Care Med 2016;44:308.

55. Carley S, Beardsell I, May N et al. Social-media-enabled learning in emergency medicine: a case study of the growth, engagement and impact of a free open access medical education blog. Postgrad Med J 2018;94:92-6.

56. Schaufeli WB. Burnout: A Short Socio-Cultural History. In: Neckel S, Schaffner AK, Wagner G, editors. Burnout, Fatigue, Exhaustion. Cham: Springer International Publishing; 2017. p. 105-27.

57. Schaufeli WB, Leiter MP, Maslach C. Burnout: 35 years of research and practice. Career Dev Int 2009;14:204-20.

58. National Academies of Sciences E, Medicine. Taking Action Against Clinician Burnout: A Systems Approach to Professional Wellbeing. Washington DC: National Academies Press; 2019. 334 p.

59. Vercio C, Loo LK, Green M, et al. Shifting Focus from Burnout and Wellness toward Individual and Organizational Resilience. Teach Learn Med 2021:1-9. [Epub ahead of print]. doi: 10.1080/10401334.2021.1879651.

Cite this article as: Crowe L, Young J, Turner MJ. What is the prevalence and risk factors of burnout among pediatric intensive care staff (PICU)? A review. Transl Pediatr 2021;10(10):28252835. doi: 10.21037/tp-20-400 